University of Wollongong

Research Online

Faculty of Engineering - Papers (Archive)

Faculty of Engineering and Information

Sciences

$1-1-2006$

\title{
Contact state determination for robotic-based cylindrical pair height adjustment under dynamic conditions
}

Prasan I. De Waas Tilakaratna

Monash University

Bijan Shirinzadeh

Monash University

Gursel Alici

University of Wollongong, gursel@uow.edu.au

Follow this and additional works at: https://ro.uow.edu.au/engpapers

Part of the Engineering Commons

https://ro.uow.edu.au/engpapers/3510

\section{Recommended Citation}

De Waas Tilakaratna, Prasan I.; Shirinzadeh, Bijan; and Alici, Gursel: Contact state determination for robotic-based cylindrical pair height adjustment under dynamic conditions 2006, 78-83.

https://ro.uow.edu.au/engpapers/3510

Research Online is the open access institutional repository for the University of Wollongong. For further information contact the UOW Library: research-pubs@uow.edu.au 


\title{
Contact State Determination for Robotic-Based Cylindrical Pair Height Adjustment under Dynamic Conditions
}

\author{
P.I. De Waas Tilakaratna*, B. Shirinzadeh* and G. Alici** \\ * Monash University/Department of Mechanical Engineering, Melbourne, Australia \\ ** University of Wollongong / School of Mechanical, Materials and Mechatronics Engineering, Wollongong, Australia \\ prasan@eng.monash.edu.au,Bijan.Shirinzadeh@eng.monash.edu.au,gursel@uow.edu.au
}

\begin{abstract}
This paper investigates fine motion strategies associated with the height adjustment manipulations of a cylindrical pair which can be utilised in dynamic assembly and reconfigurable workholding systems. The outer link of the cylindrical pair is held by the end-effector of a $6 \mathrm{DOF}$ serial articulated robot. The environment which holds the inner link can be dynamic. Formulations capable of identifying the type of contact between the links and parameters vital for control purposes based on the force and torque data provided by a wrist mounted $F / T$ sensor are established for a generic spatial case. Experimental results indicate that the established methodology is effective in determining the state of contact for the height adjustment operation.
\end{abstract}

\section{INTRODUCTION}

Lower pairs form the fundamental building blocks of many mechanisms and most assembly operations. For example, general manufacturing/assembly tasks such as position adjustment of a sleeve in a shaft prior to a welding operation are described by a cylindrical pair. Likewise, adjustment of a hinge is described by a revolute pair, where as tightening a nut and a bolt is represented by a screw pair.

The study presented in this paper is a part of an ongoing project on the height adjustment of a cylindrical pair with a dynamic inner link. Outer link attached to an articulated robot manipulator is force controlled in the compliant directions and position controlled in the direction of the height adjustment. The inner link is attached to a Cartesian manipulator with three translational DOF. The Experimental setup is depicted in Figure 1.

A number of studies on manipulations related to lower pairs are available in the literature $[1,4-6,8]$. Mason $[3,4]$ investigates pushing manipulations on a hinge with the assumption of negligible inertial forces where a numerical procedure is utilised to determine the object's instantaneous rotation center. Peshkin and Sanderson [5, 6] present the concept of Configuration Maps to determine the sequence of operations necessary to align a workpiece that slides. The effectiveness of the technique has been consolidated by aligning workpieces with automated design of a class of passive part-feeders consisting of multiple sequential fences across a conveyor belt.

Reconfigurable workholding systems utilise mechanisms for reconfiguring or rearranging elements to form layouts which would position and hold different workpieces for further machining, assembly, etc [9]. The basic structure of these mechanisms is a cylindrical pair [[8] that is adjusted accordingly depending on the geometrical characteristics of the workpiece to be held by the mechanisms. The adjustment of a cylindrical pair is briefly investigated under static conditions in Shen (1999) [7], i.e. with a fixed inner link. A basic dynamic model is developed for a non-moving environment [8] although its relevance to the manipulation is not fully investigated.

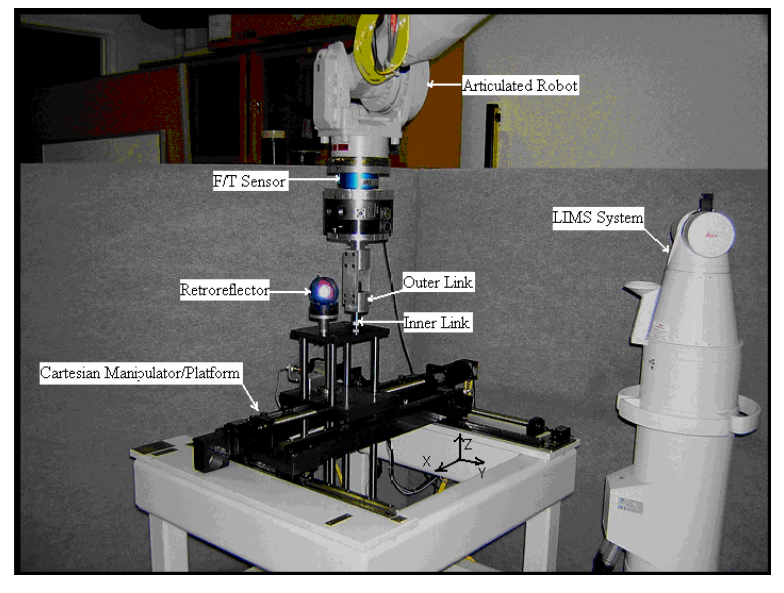

Figure 1. Experimental setup

During the height adjustment of a cylindrical pair with a loose running fit, the following contact types between the inner and the outer links are possible.
1. Null Contact
2. Single-Point Contact
3. Line Contact
4. Two-Point Contact

Among the above four contact types, the most preferred contact scenario would be the Null Contact type, although highly unfeasible in reality for parts with low clearances. Appropriate conditions for the height adjustment of a cylindrical pair can be maintained only by identifying the contact state, i.e. contact type and relevant contact forces, and subsequently utilising this knowledge to maintain minimum contact forces between the links particularly when the inner link is dynamic, i.e. non-stationary. In this investigation, $\mathrm{F} / \mathrm{T}$ sensor data is utilised to identify the contact state between the links. 
A comprehensive dynamic analysis associated with the adjustment of a cylindrical lower pair for a generic spatial case is developed, and its relevance to the adjustment operation is investigated. The underlying reasons for analysing the dynamics here is to identify the type of contact and pertinent forces that exists between the outer and the inner link in order to perform the appropriate translation and orientation adjustment. Next, we elaborate on two contact state identification techniques known as the force based approach and the $d_{\text {value }}$ based approach. Then, it is argued that the $\mathrm{d}_{\mathrm{value}}$ approach should be utilised to identify the type of contact and relevant parameters for control purposes, and if the type of contact is two point, it is necessary to employ the force based approach. Finally, experimental results which further consolidate the established methodology are presented.

\section{DYNAMIC ANALYSIS}

An F/T sensor mounted to the robot wrist provides necessary force feedback for control purposes. In this section, the objective is to establish relationships between pertinent geometric parameters, contact forces and acquired $\mathrm{F} / \mathrm{T}$ sensor data so that the contact state between the links, i.e. type of contact and contact forces, can be established.

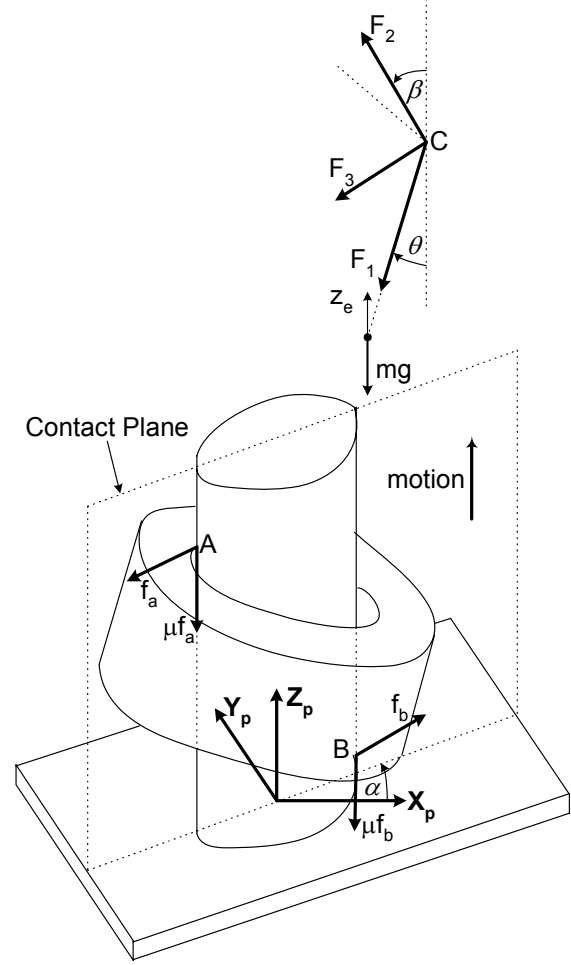

Figure 2. Cylindrical pair with two point contact

Consider a setup consisting of a cylindrical pair outer link attached to a 6DOF articulated manipulator and the inner link attached to a dynamic environment with three orthogonal translational DOF. As the clearance between the inner and the outer links is considered to be small, in a two point contact situation, it is appropriate to assume that the contact points and contact forces to be on a plane coincident with diameters of the inner and the outer links as depicted in Fig. 2. This plane also contains the $Z_{p}$-axis and is referred to as the contact plane. The contact plane contains the origin of the frame $\mathrm{F}_{1}-\mathrm{F}_{2}-\mathrm{F}_{3}$ and axis $\mathrm{F}_{1}$ at all times. The misalignment between the outer link and the inner link on the contact plane is $\theta$. The angle between the contact plane and the reference frame axis $X_{p}$ attached to the outer link is $\alpha$, where the sense is positive when rotated about the axis $Z_{\mathrm{p}}$. The angle between the axis $F_{2}$ and the contact plane is $\beta$. Friction forces due to relative translational and rotational motion of the links are always parallel or perpendicular to the Z-axis.

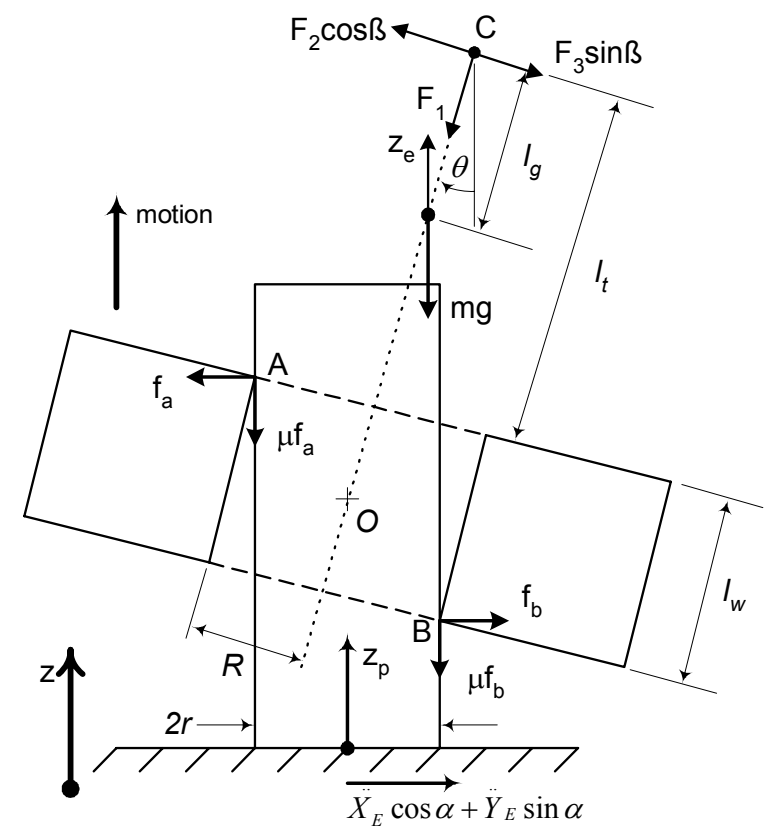

Figure 3. Forces on contact plane

Tangential forces at contact points due to permitted rotation of the outer link about its longitudinal axis are considered in this study. The forces at contact points - i.e. normal force, friction due to sliding and tangential friction due to rotation - are deemed to be orthogonal to each other. Forces on the contact plane are depicted in Fig. 3. Only $Z$ and $Z_{p}$ are specified as the reference axes due to possible rotation of the contact plane about the axis $Z_{p}$.

The effective acceleration on the contact plane as depicted in Fig.3 due to $\ddot{X}_{E}$ and $\ddot{Y}_{E}$ is $\left(\ddot{X}_{E} \cos \alpha+\ddot{Y}_{E} \sin \alpha\right)$, where $\ddot{X}_{E}$ and $\ddot{Y}_{E}$ are the acceleration components of the end-effector and are considered to be similar to the accelerations of the Cartesian manipulator along the respective directions. By employing D'Alembert's principle, the following equations are established.

$f_{a}=\frac{1}{2 \mu}\left[\left(F_{2} \cos \beta-F_{3} \sin \beta\right)(\sin \theta-\mu \cos \theta)-F_{1}(\cos \theta+\mu \sin \theta)\right.$

$\left.-\mu m\left(\ddot{X}_{p} \cos \alpha+\ddot{Y}_{p} \sin \alpha\right)-m g-m \ddot{Z}_{p}-m \ddot{Z}_{e}\right]$

$f_{b}=\frac{1}{2 \mu}\left[\left(F_{2} \cos \beta-F_{3} \sin \beta\right)(\sin \theta+\mu \cos \theta)-F_{1}(\cos \theta-\mu \sin \theta)\right.$

$\left.+\mu m\left(\ddot{X}_{p} \cos \alpha+\ddot{Y}_{p} \sin \alpha\right)-m g-m \ddot{Z}_{p}-m \ddot{Z}_{e}\right]$ 
$F_{1}, F_{2}, F_{3}, M_{1}, M_{2}$ and $M_{3}$ are measured with the aid of the wrist mounted $\mathrm{F} / \mathrm{T}$ sensor. Estimation of $\theta$ via orientation feedback of the robot or by other external means such as laser interferometry-based measurements is impractical due to robot compliance. Consequently, it is of immense significance if a set value of $\theta$ could be utilised in the above developed force relations. Given that the clearance between the links is small, the assumption of $\theta \approx$ 0 is evident to be advantageous.

For near zero values of $\theta$, the approximations $\sin \theta \approx 0$ and $\cos \theta \approx 1$ can be employed. By substituting these approximations in the above relations, the following expressions for the two possible contact forces are derived.

$$
\begin{aligned}
& f_{a}=\frac{1}{2 \mu}\left[\mu F_{3} \sin \beta-\mu F_{2} \cos \beta-F_{1}-\mu m\left(\ddot{X}_{p} \cos \alpha+\ddot{Y}_{p} \sin \alpha\right)\right. \\
& \left.-m g-m \ddot{Z}_{p}-m \ddot{z}_{e}\right]
\end{aligned}
$$$$
f_{b}=\frac{1}{2 \mu}\left[\mu F_{2} \cos \beta-\mu F_{3} \sin \beta-F_{1}+\mu m\left(\ddot{X}_{p} \cos \alpha+\ddot{Y}_{p} \sin \alpha\right)\right.
$$$$
\left.-m g-m \ddot{Z}{ }_{p}-m \ddot{z}_{e}\right]
$$

Eqs. (3) and (4) describe contact forces exerted between the links. Contact force deviations due to employing the assumption $\theta \approx 0$ when estimating contact forces have been shown to be within acceptable limits [2].

\section{Contact State Determination - Force Based APPROACH}

The contact state between the links and the control action required to minimise contact forces cannot be established solely with force data obtained via the wrist mounted $\mathrm{F} / \mathrm{T}$ sensor. As the clearance between the links is small, the resulting misalignment $\theta$ is minute, and consequently impossible to measure predominantly due to the non-collocated nature of the position feedback sensors, compliance, and drive train backlash of the articulated manipulator. The four possible contact types and relevant control parameters are determined as described below.

Null Contact: This is the most favourable condition for the height adjustment operation. In such circumstances, it is absolutely unnecessary for the controller to take any action except the height adjustment in the unconstrained direction. Null-contact is simply identified by the condition $\mathrm{f}_{\mathrm{a}} \approx 0$ and $\mathrm{f}_{\mathrm{b}} \approx 0$. The values of $\mathrm{f}_{\mathrm{a}}$ and $\mathrm{f}_{\mathrm{b}}$ are evaluated from (3) and (4).

Single-Point Contact: In a single-point contact situation, either point A or point B as indicated in Fig. 3, is in contact with the inner link. In this case, the force controller performs an appropriate translational adjustment along the compliant directions so as to minimise the contact force between the links. In single point contact, either $\mathrm{f}_{\mathrm{a}} \approx 0$ or $\mathrm{f}_{\mathrm{b}} \approx 0$. The extent of the controller action, which is a translational adjustment, is determined depending on the value and the sense of $f_{a}$ or $\mathrm{f}_{\mathrm{b}}$.
Two-Point Contact: Two-point contact is the case when both $\mathrm{f}_{\mathrm{a}}$ and $\mathrm{f}_{b}$ are non-zero. In such situation, it is important to determine the sense of the misalignment angle $\theta$ prior to the controller action.

In a two-point contact situation, information concerning the sense of the misalignment $\theta$ is essential to perform appropriate orientation adjustment manipulations. In order to identify the sense of the misalignment, estimated forces are utilised to evaluate moments for both positive and negative misalignments, and these moments are subsequently compared with the measured moments. Let $M_{1}^{c}(+\theta), M_{2}^{c}(+\theta)$ and $M_{3}^{c}(+\theta)$ be the calculated moments about axes $\mathrm{F}_{1}, \mathrm{~F}_{2}$ and $\mathrm{F}_{3}$, respectively, due to a positive misalignment, determined by manipulating the known contact forces, friction, and gravitational forces. Moments corresponding to a positive misalignment are calculated using (5).

$M_{1,2,3}^{c}(+\theta)=\sum r(+\theta) \times F$

By using (5), the following relations are established.

$M_{1}^{c}(+\theta)=-\left(f_{a}+f_{b}\right) \mu R$

$M_{2}^{c}(+\theta)=f_{a}\left\{l_{t} \sin \beta+\mu l_{t} \cos \beta-\mu R \sin \beta\right\}$

$+f_{b}\left\{\mu R \sin \beta-\left(l_{t}+l_{w}\right) \sin \beta-\left(l_{t}+l_{w}\right) \mu \cos \beta\right\}$

$M_{3}^{c}(+\theta)=f_{a}\left\{l_{t} \cos \beta-\mu R \cos \beta-\mu l_{t} \sin \beta\right\}$

$+f_{b}\left\{\mu R \cos \beta-\left(l_{t}+l_{w}\right) \cos \beta+\left(l_{t}+l_{w}\right) \mu \sin \beta\right\}$

Let $M_{1}^{c}(-\theta), M_{2}^{c}(-\theta)$ and $M_{3}^{c}(-\theta)$ be the calculated moments due to a negative misalignment and using a similar procedure as for the positive misalignment scenario, the following moment relations are established.

$M_{1}^{c}(-\theta)=-\left(f_{a}+f_{b}\right) \mu R$

$M_{2}^{c}(-\theta)=f_{a}\left\{\mu\left(l_{t}+l_{w}\right) \cos \beta+\left(l_{t}+l_{w}-\mu R\right) \sin \beta\right\}$

$+f_{b}\left\{\mu R \sin \beta-\mu l_{t} \cos \beta-l_{t} \sin \beta\right\}$

$M_{3}^{c}(-\theta)=f_{a}\left\{\left(l_{t}+l_{w}-\mu R\right) \cos \beta-\mu\left(l_{t}+l_{w}\right) \sin \beta\right\}$

$+f_{b}\left\{\mu l_{t} \sin \beta+\mu R \cos \beta-l_{t} \cos \beta\right\}$

Although (3) and (4) evaluate the contact forces, they do not provide information regarding the sense of the misalignment angle $\theta$. In order to verify the sense of the misalignment, actual moment components $\mathrm{M}_{2}$ and $\mathrm{M}_{3}$ measured by the wrist mounted $\mathrm{F} / \mathrm{T}$ sensor and the calculated moment components $M_{2}^{c}(+\theta), \quad M_{2}^{c}(-\theta)$, $M_{3}^{c}(+\theta)$ and $M_{3}^{c}(-\theta)$ are compared accordingly as shown below. A comparison between $M_{1}^{c}(+\theta)$ and $M_{1}^{c}(-\theta)$ is redundant as both of these moment components are due to the tangential friction forces and are independent of the misalignment sense. 
For positive misalignment:

$$
\begin{aligned}
& M_{2} \approx M_{2}^{c}(+\theta) \\
& M_{3} \approx M_{3}^{c}(+\theta)
\end{aligned}
$$

Similarly, for negative misalignment:

$$
M_{2} \approx M_{2}^{c}(-\theta)
$$

$$
M_{3} \approx M_{3}^{c}(-\theta)
$$

During two point contact, the controller action consists of a translational as well as a rotational adjustment.

Line Contact: Line contact can be considered as a variant of single point contact where the contact force is arbitrarily distributed along the line of contact between the links. The force distribution depends on parameters such as the misalignment sense prior to contact, surface texture and system compliance. However, it is important to note that the resultant contact force on the contact plane and the friction force in the z-direction are similar to that of a single point contact situation. Consequently, the controller action is identical to the single point contact case.

\section{Contact State Determination $-\mathrm{d}_{\text {VAlue }}$ BASED APPROACH}

The methodology established to determine the contact state between a cylindrical pair in the preceding section involves estimating the contact force values $f_{a}$ and $f_{b}$. Rather than observing the contact forces $f_{a}$ and $f_{b}$ in order to determine the contact state, the value of $d$ depicted in Fig. 4 is utilised to distinguish between different contact types. Herein, we refer to this value as the " $d_{\text {value }}$ " [1].

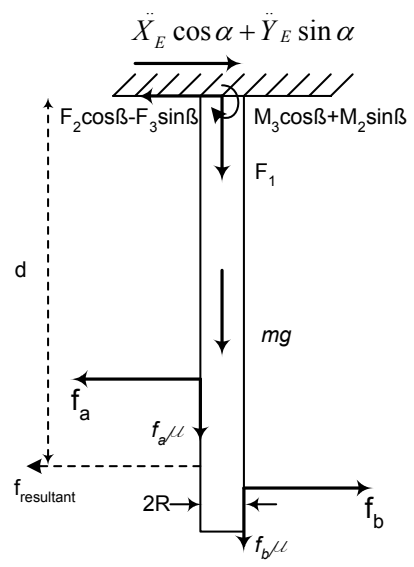

Figure 4. Beam representation of the outer link

Fig. 4 depicts the effective beam representation of the outer link for a spatial analysis under dynamic environment conditions. Essentially, the $\mathrm{d}_{\mathrm{value}}$ is the distance from the origin to the line of action of the resultant force of $f_{a}$ and $f_{b}$. From a momentum balance, the following relations are established. $d_{\text {value }}=\mu R+\frac{M_{R}}{F_{R}}$

Where,

$F_{R}=F_{2} \cos \beta-F_{3} \sin \beta+m\left(\ddot{X}_{E} \cos \alpha+\ddot{Y}_{E} \sin \alpha\right) l_{g}$

$M_{R}=M_{3} \cos \beta+M_{2} \sin \beta+m\left(\ddot{X}_{E} \cos \alpha+\ddot{Y}_{E} \sin \alpha\right) l_{g}$

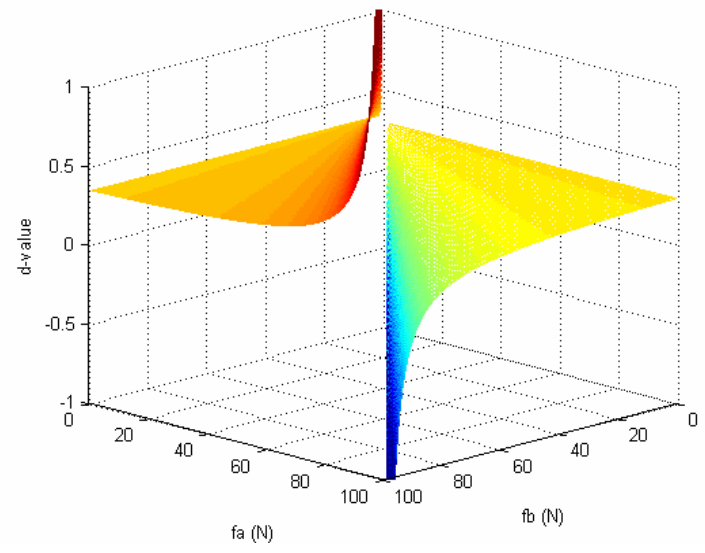

Figure 5. Variation of the $d_{\text {value }}$ with contact forces $\mathrm{f}_{\mathrm{a}}$ and $\mathrm{f}_{\mathrm{b}}$

Fig. 5 depicts the variation of the $d_{\text {value }}$ for a positive misalignment $\theta$ as a function of contact forces $f_{a}$ and $f_{b}$. The $d_{\text {value }}$ at $f_{a}=0$ and $f_{b}=0$ corresponds to 0.337 and 0.287 , respectively, as the distances from the origin point of the $\mathrm{F} / \mathrm{T}$ sensor to the upper and lower circumferential edges of the outer link are $287 \mathrm{~mm}$ and $337 \mathrm{~mm}$. Identification of contact state using the $\mathrm{d}_{\text {value }}$ is presented in the remainder of this section.

Null Contact: In a null contact situation, both $\mathrm{M}_{\mathrm{R}}$ and $F_{R}$ described by (17) and (18), respectively, are zero. Rather than utilising the $\mathrm{d}_{\text {value }}$ which approaches a singularity under such conditions, we identify a null contact type by realising that $\mathrm{M}_{\mathrm{R}}$ and $\mathrm{F}_{\mathrm{R}}$ are zero.

Single-Point Contact: The $\mathrm{d}_{\mathrm{value}}$ can be successfully utilised to identify a single point contact situation. As evident in Fig.5, during single point contact, $\mathrm{d}_{\text {value }}=$ 0.2874 or $\mathrm{d}_{\text {value }}=0.3374$. One can intuitively deduce the $d_{\text {value }}$ to be either $l_{t}$ or $l_{t}+1_{w}$ since the contact points lie on one of the two outer link circumferential edges. The control action is dependant on the magnitude and the sense of $\mathrm{F}_{\mathrm{R}}$ given by (17).

Line Contact: In a line contact situation, the resultant force due to the force exerted between the links along the contact line is located between the two circumferential edges of the outer-link. Therefore, for line contact, $l_{t}<$ $d_{\text {value }}<1_{t}+1_{w}$. Control action is similar to that of the single point contact situation.

Two-Point Contact: As indicated earlier, in a two-point contact situation, neither $f_{a}$ nor $f_{b}$ is zero. As evident in Fig. 5 , in such a circumstances, $d_{\text {value }}<1_{t}$ and $d_{\text {value }}>1_{t}+1_{w}$. When $\mathrm{f}_{\mathrm{a}}$ and $\mathrm{f}_{\mathrm{b}}$ are nonzero, $\mathrm{d}_{\text {value }}<0.2874$ or $\mathrm{d}_{\text {value }}>$ 0.3374 . One can also establish the relevant range of the $\mathrm{d}_{\text {value }}$ by realising the fact that the line of action of the resultant force must lie outside the region between the contact forces. It is important to note that the sense of the 
misalignment cannot be determined using this technique. Thus, in a two point contact situation, it is necessary to employ the force based technique in order to establish required parameters for control purposes. The relevant controller actions necessary to minimise contact forces between the links are identical to that of described in the previous section.

\section{EXPERIMENTAL RESUlTS}

The nominal friction coefficient has been experimentally estimated to be 0.145 . From (3) and (4) it is evident that the force based approach for contact state determination is highly sensitive to friction at the contact point. On the other hand, from (16), it is clear that the $\mathrm{d}_{\text {value }}$ is less sensitive to friction as the effect due to the term $\mu R$ is minor. Hence, it is argued that $d_{\text {value }}$ is the most suitable to identify the state of contact between a cylindrical pair. However, if the type of contact is two point, the force based approach must be employed to determine the sense of the misalignment and relevant parameters for control purposes.

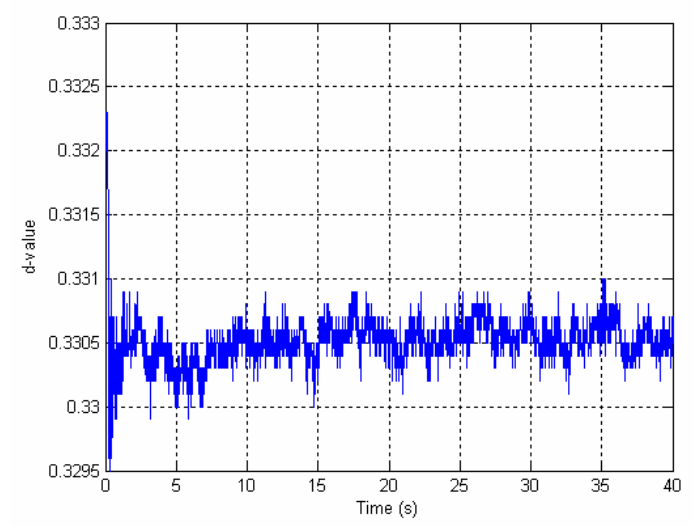

Figure 6. Behaviour of $d_{\text {value }}$ for single point contact

The experimental setup shown in Fig. 1 includes a Motoman SK-120 articulated robot manipulator, a JR3 $\mathrm{F} / \mathrm{T}$ sensor attached to the wrist where the outer link and the inner link of the cylindrical pair are attached to the F/T sensor and a Cartesian manipulator, respectively. Different contact types were deliberately introduced by the robot manipulator. During the experiments, the height adjustment was performed by maintaining the introduced configurations whilst acquiring force/moment data. The acquired data was processed, and subsequently utilised in the formulations in order to determine the state of contact using the proposed techniques.

Fig. 6 depicts the behaviour of the $d_{\text {value }}$ during single point contact. The mean and the standard deviation of the $\mathrm{d}_{\text {value }}$ are 0.3305 and 0.0002 , respectively. There is a $95 \%$ confidence level in obtaining a $\mathrm{d}_{\text {value }}$ of $0.331 \pm 0.000$. However, for the links utilised in the experiments, the desired $\mathrm{d}_{\text {value }}$ is 0.337 . Observed deviations are due to geometric uncertainties, parameter variations, approximations, etc.

Behaviour of the $d_{\text {value }}$ for an adjustment during line contact is depicted in Fig. 7. The mean value and the standard deviation of the relevant $d_{\text {value }}$ are observed to be 0.316 and 0.0003 , respectively. The relevant $95 \%$ confidence level is $0.316 \pm 0.000$. This estimate is well within the theoretically predicted range $0.287-0.337$.

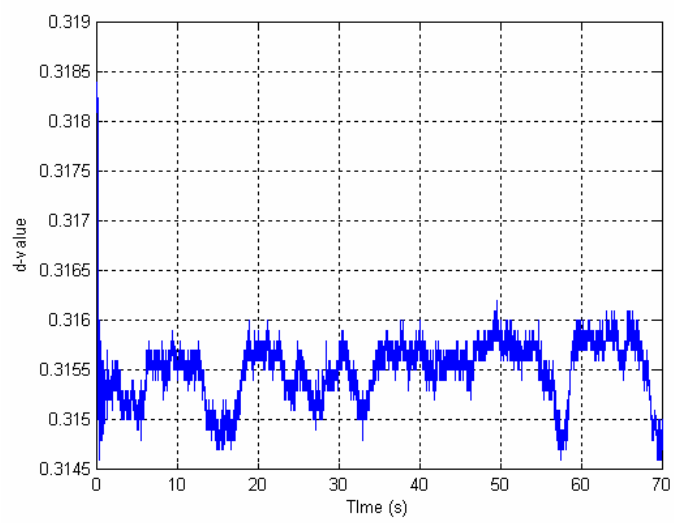

Figure 7. Behaviour of $d_{\text {value }}$ for line contact

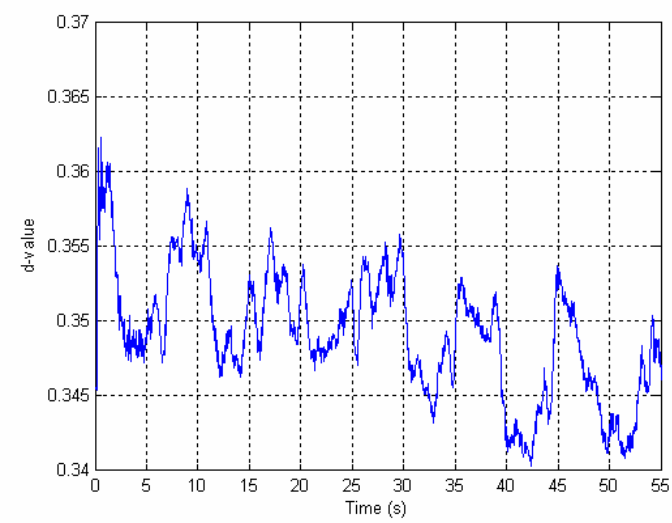

Figure 8. Behaviour of $\mathrm{d}_{\text {value }}$ for two point contact

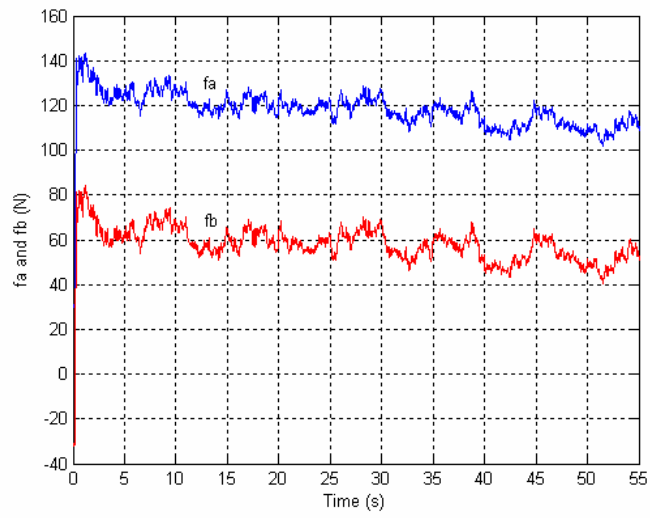

Figure 9. Contact forces during two point contact

The $\mathrm{d}_{\text {value }}$ corresponding to a two point contact situation is depicted in Fig.8. The 95\% confidence range for the estimated $\mathrm{d}_{\text {value }}$ is $0.349 \pm 0.000$. Although the estimated $\mathrm{d}_{\text {value }}$ conforms to the theoretical prediction, $\mathrm{d}_{\mathrm{value}}$ alone do not provide adequate information regarding the sense of the misalignment and parameters necessary for control purposes. Hence, contact forces are evaluated using (3) 
and (4), and a moment comparison described in Section III is employed to determine the sense of the misalignment.

As depicted in Fig. 9, the mean contact forces $f_{a}$ and $f_{b}$ for a two-point contact adjustment have been estimated to be $117.6 \mathrm{~N}$ and $57.4 \mathrm{~N}$, respectively.

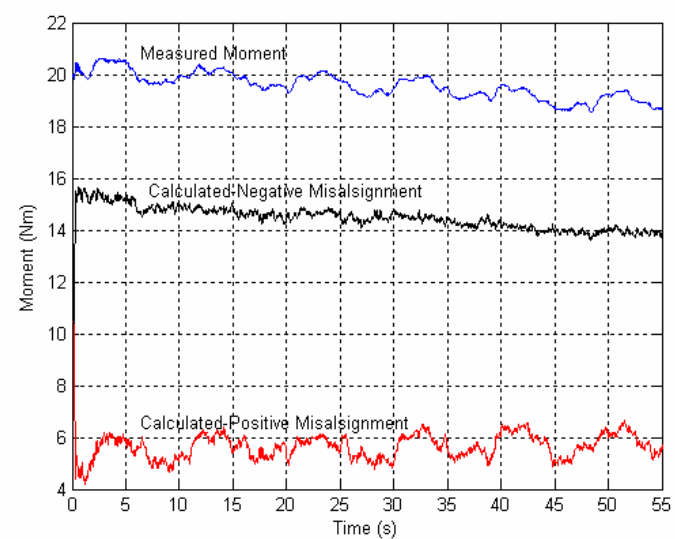

Figure 10. Moment comparison for two point contact

A negative misalignment was maintained during experiments. Calculated moments and the measured moment are shown in Fig. 10. Ideally, the calculated moment for a negative misalignment and the measured moment should be similar. However, a difference of approximately $5 \mathrm{Nm}$ exists between the two moments which is primarily due to friction coefficient variations at the contact point.

Experimental results clearly consolidate the effectiveness of the established methodology for contact state identification. The sensitivity of the force based approach to friction is further proven by the discrepancies evident in the calculated moments depicted in Fig. 10.

\section{CONCLUSION}

A methodology to identify the contact state for roboticbased height adjustment of a cylindrical pair is presented. The technique is based on maintaining minimum contact forces between the links. Dynamic formulations capable of extracting parameters for control purposes and to determine the type of contact between the links are derived from a spatial point of view. Two techniques known as the force-based approach and the $d_{\text {value }}$-based approach have been established for contact state identification. The $\mathrm{d}_{\text {value }}$-based approach is utilised to determine the type of contact of a cylindrical pair subjected to robot-based height adjustment, and if the type of contact is two-point, the force-based approach is subsequently employed to extract necessary parameters for control purposes. Experimental results prove the feasibility and the effectiveness of the proposed methodology for contact state determination.

\section{ACKNOWLEDGMENT}

This study has been partly supported by Australian Research Council (ARC) and Monash Research Fund.

\section{REFERENCES}

[1] P. De Waas Tilakaratna, B. Shirinzadeh, and G. Alici, "Contact Type Determination for Cylindrical Pair Adjustment," Proceeding of the IEEE/ASME International Conference on Advance Intelligent Mechatronics, Kobe, Japan, Vol 1, pp 326-331, 2003.

[2] P. De Waas Tilakaratna, B. Shirinzadeh, and G. Alici, "Experimental Investigation of 2D Cylindrical Pair Height Adjustment in a Static Environment," Proceedings of the 3rd IFAC Symposium on Mechatronic Systems, Sydney, Australia, Vol 1, pp 663-668, 2004.

[3] M. T. Mason, "Mechanics and Planning of Manipulator Pushing Operations," The International Journal of Robotics Research, vol. 5, pp. 53-71, 1986.

[4] M. T. Mason, "Two Graphical Methods for Planar Contact Problems," Proceedings of the IEEE/RSJ International Workshop on Intelligent Robots and Systems, Osaka, Japan, Vol 2, pp 443-448, 1991.

[5] M. A. Peshkin and A. C. Sanderson, "The Motion of a Push Sliding Workpiece," IEEE Journal of Robotics and Automation, vol. 4, pp. 569-598, 1988a.

[6] M. A. Peshkin and A. C. Sanderson, "Planning Robotic Manipulations Strategies for Workpieces," IEEE Journal of Robotics and Automation, vol. 4, pp. 523-531, $1988 \mathrm{~b}$.

[7] Y. L. Shen, "A Theoretical and Computational Study of Robotic Manipulation Operations on Passive Mechanisms," Masters Thesis, Department of Mechanical Engineering, Monash University, Melbourne, 1999.

[8] Y. L. Shen and B. Shirinzadeh, "Dynamic Analysis of Reconfigurable Fixture Construction by a Manipulator," Robotics and Computer Integrated Manufacturing, vol. 17, pp. 367-377, 2001.

[9] B. Shirinzadeh, "Flexible Fixturing for Workpiece Positioning and Constraining," Assembly Automation, vol. 22, pp. 112-120, 2002. 